\title{
ESTIMATIVA DA ÁREA DE MANDIOCA INDUSTRIAL NA REGIÃO DE PARANAVAÍ, ESTADO DO PARANÁ, POR MEIO DO SENSOR LANDSAT TM 5
}

\author{
ESTIMATION OF THE CASSAVA AREA FOR STARCH PRODUCTION \\ IN PARANAVAÍ REGION, PARANÁ STATE, BRAZIL, THROUGH \\ THE SENSOR LANDSAT TM 5
}

\begin{abstract}
ESTIMATION DE LA SURFACE DE MANIOC POUR LA PRODUCTION
DE FECULE DANS LA REGION DE PARANAVAI, ÉTAT DU PARANA, BRESIL, AVEC IMAGES DU SENSEUR LANDSAT TM 5
\end{abstract}

\author{
Brychtn Ribeiro de Vasconcelos - Universidade Católica Dom Bosco - Campo Grande - \\ Mato Grosso do Sul - Brasil \\ brychtn@gmail.com \\ Olivier François Vilpoux - Universidade Católica Dom Bosco - Campo Grande - \\ Mato Grosso do Sul - Brasil \\ vilpoux@ucdb.br \\ Antonio Conceição Paranhos Filho - Universidade Federal de Mato Grosso do Sul - \\ Campo Grande - Mato Grosso do Sul - Brasil \\ antonio.paranhos@pq.cnpq.br
}

\section{Resumo}

0 artigo avalia o uso de geotecnologias como apoio ao setor de mandioca industrial para produção de fécula. A pesquisa verificou a possibilidade de identificar as áreas plantadas com mandioca industrial no Paraná, na microrregião de Paranavaí, maior região brasileira de produção de fécula, por meio das imagens do sensor Thematic Mapper do satélite Landsat 5. A obtenção de informações sobre a área plantada de mandioca deve reduzir a incerteza no setor feculeiro da região e diminuir as flutuações do preço de fécula. A avaliação foi dividida em três etapas: localização de 85 áreas de plantio e elaboração de uma chave de identificação; verificação das previsões e validação da chave de identificação; avaliação da área plantada em três municípios e comparação com os dados do IBGE. Os resultados apresentaram eficiência de $95 \%$ na identificação das áreas com mandioca. A previsão limita-se a áreas superiores a 2 ha, 0 que não apresenta problema para a microrregião de Paranavaí, mas pode ser limitante em outras regiões feculeiras, como o Oeste do Paraná. Mesmo assim, 0 sensoriamento remoto é uma ferramenta de grande utilidade para a previsão de safra de mandioca industrial. Ela poderá complementar os dados do IBGE, que foram muito diferentes.

Palavras-chave: sensoriamento remoto, fécula, estabilidade de preço, incerteza.

\section{Abstract}

The paper evaluates the use of geotechnology supporting the evaluation of cassava harvest for starch production. The research examined the possibility of identifying areas planted with cassava for industrial use in Paraná State, in the micro region of Paranavai, largest Brazilian region for starch production, through the use of images from the Thematic Mapper sensor onboard the Landsat 5. Obtaining information on the area planted with cassava should reduce uncertainty of the cassava starch industry in the region and reduce fluctuations in the price of starch. The evaluation was divided into three steps: location of 85 planting areas and development of an identification key; verification of the forecasts and validation of the 
identification key; evaluation of the planted area in three municipalities and comparison with data from the IBGE. The results showed an efficiency of $95 \%$ in identifying the areas with cassava. The forecast is limited to areas greater than 2 ha, which presents no problem for the micro region of Paranavai, but can be limiting in other regions, such as the West of Paraná. Nevertheless, remote sensing is a valuable tool for predicting production of industrial cassava. It could complement the IBGE data, which were very different.

Keywords: remote sensing, cassava starch, price stability, uncertainty.

\section{Résumé}

L'article évalue l'utilisation de géotechnologies pour prévoir la production de manioc destiné à la fécule. La recherche étudie la possibilité d'identifier les superficies plantées en manioc industriel dans l'état du Paraná, dans la région de Paranavaí, la plus importante pour la production brésilienne de fécule. Des images du capteur Thematic Mapper, du satellite Landsat 5, ont été utilisées. Obtenir des informations sur la superficie plantée en manioc devrait réduire l'incertitude dans le secteur féculier de la région et diminuer les variations du prix de l'amidon. L'analyse a été divisée en trois étapes: localisation de 85 champs de manioc et développement d'une clé d'identification ; vérification des prévisions et validation de la clé d'identification ; évaluation de la surface total plantée en manioc dans trois villes et comparaison avec les données IBGE. Les résultats indiquent une efficacité de $95 \%$ dans l'identification des champs de manioc. La prévision est limitée à des surfaces supérieures à 2 ha, ce qui ne pose aucun problème pour la région de Paranavaí, mais peut être un facteur limitant dans d'autres régions, comme l'Ouest du Paraná. Néanmoins, la télédétection est un outil précieux pour estimer les surfaces plantées en manioc et compléter les données de l'IBGE, qui étaient très différentes.

Mots-clés: télédétection, fécule de manioc, stabilisation des prix, incertitude.

\section{Introdução}

A mandioca (Manihot esculenta Crantz) é uma planta amazônica e já era utilizada pelos índios. Após a chegada dos portugueses no Brasil, ela foi disseminada na África e Ásia. Segundo a FAO (FAOSTAT, 2012), em 2010, os principais países produtores de mandioca eram a Nigéria, a República Democrática do Congo, o Brasil, a Indonésia e a Tailândia.

A mandioca não só é utilizada por milhões de pessoas na alimentação humana e animal, como serve também de matéria-prima na fabricação de vários produtos e subprodutos. Os principais são a farinha, consumida principalmente no Brasil e na África, e a fécula, ou amido, produzida essencialmente na Ásia - com a Tailândia como principal produtor - e no Brasil.

A produção nacional de fécula de mandioca ocorre essencialmente nos Estados da região Sudeste, Sul e Centro-Oeste. O Estado do Paraná é o principal produtor de fécula, com $70 \%$ da produção, seguido de Mato Grosso do Sul e de São Paulo. A indústria de processamento de fécula de mandioca é mais moderna do que a de farinha. O amido é considerado uma commodity e a fécula de mandioca é o principal amido exportado no mundo, com dominação da Tailândia (Vilpoux, 2008, p. 32). 
Para Vilpoux (2010, p. 128), o principal gargalo do setor de amido de mandioca no Brasil é a instabilidade da oferta, que se traduz por grandes variações de preços e afeta a rentabilidade das empresas e produtores. Períodos de grande produção, com preços baixos, desestimulam os produtores, enquanto períodos de falta de matéria-prima, com preços altos, prejudicam os mercados consumidores, com a troca por produtos alternativos, principalmente o amido de milho (Felipe et al., 2010, p. 16).

Para Goebel (2005, p. 18), a solução para esse problema passa pelo aumento das informações disponíveis sobre o cultivo de mandioca. O fornecimento de informações sobre a área plantada pode ajudar a planejar o plantio.

A previsão de safra no Brasil é feita por meio da estimativa anual da produção agrícola em escala nacional, com a utilização de questionários aplicados diretamente aos produtores ou às entidades relacionadas à atividade agrícola em cada região. A obtenção de dados confiáveis através dessa metodologia é lenta, onerosa e implica um alto grau de subjetividade. Além de que a extensão e peculiaridades regionais e sazonais do território brasileiro podem introduzir erros nos resultados (Fontana et al., 2000, p. 1).

Outra opção para a coleta de informações sobre a produção de matéria-prima agrícola é o uso de geotecnologias, como o Sensoriamento Remoto, o GPS e os Sistemas de Informações Geográficas (SIG) aplicados ao monitoramento de cultivos para a estimativa de safra. Essas tecnologias permitem o oferecimento de maior quantidade de informações, com possibilidade de dados de qualidade, com baixo custo.

O presente trabalho avaliou o potencial das geotecnologias para a estimativa de safra de mandioca. A pesquisa teve por objetivo avaliar a aplicação dessas geotecnologias na identificação e estimativa de área plantada de mandioca industrial na microrregião de Paranavaí, no Paraná, maior região de produção de fécula do Brasil.

O uso das geotecnologias deverá permitir a previsão da produção de mandioca. O agricultor, ao tomar posse dessas informações, poderá ajustar sua produção em função das previsões e das necessidades do mercado. As informações serão também úteis para a indústria planejar sua produção e incentivar os produtores. Em consequência, as informações disponíveis poderão reduzir as variações periódicas do preço da mandioca. 
Discute-se a seguir a produção de fécula de mandioca no Brasil e os impactos provocados pela falta de informação. Na sequência, são identificados os tipos de uso de geotecnologias para o levantamento de área plantada; e são apresentados a metodologia utilizada na pesquisa e os resultados da aplicação de geotecnologias na avaliação da área plantada de mandioca.

\section{Produção de fécula de mandioca no Brasil}

A mandioca é matéria-prima para inúmeros produtos, incluindo farinha, ração animal, álcool, papel, têxteis, adoçantes, alimentos preparados e produtos biodegradáveis. A utilização da mandioca como insumo industrial depende da sua disponibilidade e competitividade de preços em relação a produtos alternativos, como o milho (FAO, 2000, p. 8).

A maior parte da produção de amido de mandioca no Brasil se concentra em três Estados: Paraná, Mato Grosso do Sul e São Paulo. O Paraná lidera, com 71\% da produção nacional, seguido de Mato Grosso do Sul, com 14\%, e São Paulo, com 13\% (ABAM, 2012).

O setor produtivo de mandioca no Brasil é marcado por fortes variações periódicas, influenciadas pela oferta de produto. Felipe et al. (2010, p. 10) afirmam que o preço da mandioca no momento do plantio influencia diretamente a decisão sobre a quantidade de área a ser plantada para a safra seguinte. Segundo Vilpoux (2010, p. 137), as variações anuais elevadas de preço acontecem devido à variação na área plantada, como resposta direta aos preços praticados. Picos de preços altos seguem os de baixos, em intervalo de doze a dezoito meses, tempo equivalente ao ciclo completo de produção da cultura.

A oscilação dos preços de mandioca industrial cria uma incerteza, e surge a dificuldade em se definirem os preços futuros, estimulando o comportamento especulativo (Nogueira, 1999, p. 6). O produtor decide conforme o valor pago no momento do plantio. Ou seja, o plantio é maior nos períodos de preço alto e reduz na queda dos preços (Vilpoux, 2008, p. 27).

A importância da mandioca para as indústrias faz com que todos os elos da cadeia tendam a sofrer com a instabilidade na oferta de matéria-prima, inviabilizando a competição no mercado de amido (Cardoso; Barros, 2004, p. 1). Essa instabilidade foi definida por Cardoso (2003, p. 47) 
como um dos principais obstáculos à competição e ao desenvolvimento da cadeia nos mercados internos e externos.

Estimativas de áreas plantadas poderão monitorar toda uma região de produção de mandioca para uma indústria ou uma entidade que represente o setor e, por meio das estimativas, facilitar-se-á o planejamento da produção, evitando prejuízos futuros. A redução da incerteza é um dos fatores essenciais para se ter sucesso na gestão empresarial, e é facilitada pela obtenção de maior quantidade de informações sobre o ambiente no qual o setor está inserido (Nogueira, 1999, p. 1).

Contratos não são utilizados pelos produtores, pois eles acreditam que no futuro poderão ter prejuízos caso fiquem presos a um documento que limite sua liberdade de ação (Rinaldi et al., 2005, p. 21). Do lado dos empresários, a utilização de contrato também é rara, principalmente devido à insegurança na definição dos preços futuros (Nogueira, 1999, p. 6).

As dificuldades para se harmonizarem as relações entre produtores e feculeiros possuem outro agravante: a assimetria de informação entre produtores e indústrias. Para Cardoso (2003, p. 95), a assimetria de informação com relação aos preços pode ser reduzida investindo-se em informações sobre estimativas de área e de quantidades transacionadas nos mercados físicos regionais.

Uso de geotecnologias para levantamento de áreas plantadas

A coleta de informações sobre culturas agrícolas por meio de tecnologias espaciais se intensificou nos últimos anos. As técnicas de geoprocessamento e os Sistemas de Informações Geográficas (SIGs) permitem a obtenção de informações mais detalhadas sobre diferentes culturas. Por exemplo, o GPS (Global Positioning System) é utilizado no levantamento de campo para georreferenciar o plantio, assim como em máquinas agrícolas na agricultura de precisão, enquanto as imagens de satélites oferecem informações sobre o desenvolvimento do cultivo e estimativas de área cultivada.

\section{As geotecnologias}

O geoprocessamento é o ramo do conhecimento que faz uso das técnicas matemáticas e computacionais para tratamento de informações 
geográficas. Essa tecnologia possui custo relativamente baixo e seu potencial ainda pode ser somado ao fato de o conhecimento ser adquirido localmente. Os SIGs são os instrumentos computacionais do geoprocessamento que permitem realizar análises complexas ao incorporar dados de diversas fontes e criar bancos de dados georreferenciados (Câmara; Medeiros, 1998, p. 7).

Os SIGs são aplicados para diversas finalidades, principalmente no monitoramento de safras agrícolas. Eles permitem manipular dados extensos, de grande volume e possuidores de estruturas e inter-relacionamentos complexos (Ramirez; Souza, 2007, p. 57). Para Câmara e Medeiros (1998, p. 6), um SIG armazena a geometria e os atributos dos dados que estão georreferenciados. Um SIG pode ser utilizado, no mínimo, de três formas: como ferramenta para produção de mapas; como suporte para análise espacial de fenômenos; como um banco de dados geográficos, com funções de armazenamento e recuperação da informação.

Burrough (1986, p. 6) define o SIG como um "conjunto poderoso de ferramentas para coletar, armazenar, recuperar, transformar e visualizar dados sobre o mundo real”. A integração de vários tipos de dados (socioeconômicos, ambientais, urbanos, agrários, entre outros) em SIG permite a exploração por equipes multidisciplinares. A integração do sensoriamento remoto e de Sistemas de Informações Geográficas constitui ferramentas eficientes, com dados e resultados de boa qualidade, para pesquisas e monitoramento de áreas (Eiumnoh; Shrestha, 1999, p. 428).

Na agricultura, o sensoriamento remoto é utilizado em diversas aplicações: monitoramento agrícola, condição das culturas, previsão de safras, erosão de solos, agricultura de precisão, índice de área foliar, entre outras. Para Sanches et al. (2005, p. 83), o sensoriamento remoto constitui uma ferramenta de grande contribuição para o monitoramento da atividade agrícola. A disponibilidade de informações confiáveis sobre os tipos de culturas instaladas, área plantada e distribuição espacial dentro de uma determinada região é fundamental na tomada de decisões para o planejamento, definição de prioridades e liberação de financiamento pelos setores públicos ou privados envolvidos na agricultura. Tais informações podem ser obtidas através de métodos convencionais, envolvendo questionários aplicados diretamente aos produtores, ou através da utilização de dados de sensoriamento remoto (Sano et al., 1998, p. 179). 
Uso de geotecnologias para levantamento de área e seguimento de produção

A agricultura é a grande beneficiária das geotecnologias, essencialmente através da agricultura de precisão, que consiste no manejo estratégico de cultivos agrícolas com a aplicação de informação tecnológica e conhecimento agronômico. A agricultura de precisão trabalha com grande quantidade de dados, que são coletados e tratados visando ao desenvolvimento do poder de decisão (Santos et al., 2001, p. 1509).

As geotecnologias podem ser aplicadas em outras áreas agronômicas, tal como no levantamento de culturas agrícolas. Um sistema para estimativa da área plantada da cultura de soja, por meio das geotecnologias, foi desenvolvido e testado no Estado do Rio Grande do Sul por Rizzi e Rudorff (2005, p. 226). Esse sistema utilizou um método de classificação digital por interpretação visual das imagens, com base no comportamento espectro-temporal do plantio. A estimativa da área plantada de soja foi comparada com as estatísticas oficiais do Instituto Brasileiro de Geografia e Estatística (IBGE). Em nível estadual, foi observada uma diferença de $11,3 \%$ entre as estimativas do IBGE e das imagens Landsat. Os autores concluíram que as imagens do satélite Landsat 5 podiam ser utilizadas para o correto mapeamento da soja e para auxiliar na melhoria das estatísticas agrícolas oficiais.

Adami et al. (2005, p. 24) desenvolveram um método para estimar as áreas plantadas de café, milho e soja na cidade de Cornélio Procópio, no Estado do Paraná. Os autores utilizaram imagens de sensoriamento remoto, procedimentos de classificação de imagens, SIG e GPS. A comparação entre os resultados da pesquisa e as estimativas oficiais indicou que as melhores estimativas foram para a cultura da soja, que ocupava $32,1 \%$ da região. Para essa cultura, a diferença em relação à estimativa oficial foi de apenas 5,9\%.

Cirani e Moraes (2011, p. 561) constataram que no setor sucroalcooleiro do Estado de São Paulo, 76\% das empresas utilizavam imagens de satélite, 33\%, fotografias aéreas, e 31\%, amostragem de solo em grade (com GPS). Rudorff et al. (2004, p. 20) mapearam as lavouras de cana-de-açúcar por meio de imagens de satélites de sensoriamento remoto, em escala municipal, para todo o Estado de São Paulo - para a safra 2003/2004. Os resultados indicaram uma área total de 3,09 milhões de hectares de cana, ou $12,5 \%$ da área total do Estado. 
Na literatura internacional alguns trabalhos utilizaram geotecnologias para plantio de mandioca. Chao et al. (2007, p. 1135) avaliaram o potencial do plantio da mandioca por meio do SIG e do sensoriamento remoto. Eiumnoh e Shrestha (1999, p. 417) realizaram outro estudo sobre avaliação da área e da produção de mandioca na região nordeste da Tailândia, usando um sistema integrado de sensoriamento remoto e SIG. O objetivo do estudo foi explorar o uso da NOAA-AVHRR para mapeamento de áreas de plantação de mandioca. O estudo foi realizado para as safras 1995 e 1996 e indicou que os dados NOAA-AVHRR podem ser utilizados para mapear o plantio de mandioca em escala regional na Tailândia.

\section{Metodologia}

A técnica utilizada visa identificar a área plantada de mandioca industrial na microrregião de Paranavaí, por meio de imagens do sensor TM/Landsat 5.

\section{Geotecnologias utilizadas}

As imagens empregadas para identificar os plantios de mandioca são multiespectrais, obtidas pelo sensor TM (Thematic Mapper) do satélite Landsat 5 (Land Remote Sensing Satellite). Esse satélite, lançado em 1984, possui a bordo o sensor TM de resolução espacial de 30 metros, altura de órbita 705 km, largura de faixa imageadora de 185 km e 16 dias de duração do ciclo de cobertura para recobrimento da superfície. O satélite pertence ao Programa Landsat, composto de um conjunto de sete satélites desenvolvidos e lançados pela National Aeronautics and Space Administration - NASA (Novo, 2011, p. 135). Na Tabela 1 são apresentadas as principais características do sensor TM do satélite Landsat 5.

As imagens do sensor TM do satélite Landsat 5 utilizadas foram obtidas no website do Instituto Nacional de Pesquisas Espaciais (INPE). Foram necessárias nove imagens, correspondentes da órbita 223 e do ponto 76, obtidas no período de safra 2010/11. Foram utilizadas todas as bandas, com exceção da banda seis. As imagens compostas possuem seis bandas, e no processo de identificação dos plantios foram utilizadas composições coloridas. No final, optou-se por criar a chave de identificação apenas com a banda quatro, do infravermelho próximo. 
Tabela 1 - Principais características do sensor Thematic Mapper (TM) do satélite Landsat 5

\begin{tabular}{|c|c|c|c|c|c|c|}
\hline $\begin{array}{l}\text { Número } \\
\text { da Banda }\end{array}$ & $\begin{array}{c}\text { Intervalo e } \\
\text { Comprimento } \\
\text { de Onda }(\mu \mathrm{m})\end{array}$ & $\begin{array}{l}\text { Resposta } \\
\text { Espectral }\end{array}$ & $\begin{array}{l}\text { Resolução } \\
\text { Espacial (m) }\end{array}$ & $\begin{array}{l}\text { Resolução } \\
\text { Temporal }\end{array}$ & $\begin{array}{c}\text { Área } \\
\text { Imageada }\end{array}$ & $\begin{array}{c}\text { Resolução } \\
\text { Radiométrica }\end{array}$ \\
\hline 1 & $0.45-0.52$ & Azul-Verde & 30 & \multirow{7}{*}{16 dias } & \multirow{7}{*}{$185 \times 185 \mathrm{~km}$} & \multirow{7}{*}{8 bits } \\
\hline 2 & $0.52-0.60$ & Verde & 30 & & & \\
\hline 3 & $0.63-0.69$ & Vermelho & 30 & & & \\
\hline 4 & $0.76-0.90$ & $\begin{array}{l}\text { Infravermelho } \\
\text { Próximo }\end{array}$ & 30 & & & \\
\hline 5 & $1.55-1.75$ & $\begin{array}{l}\text { Infravermelho } \\
\text { Médio }\end{array}$ & 30 & & & \\
\hline 6 & $10.40-12.50$ & $\begin{array}{c}\text { Infravermelho } \\
\text { Termal }\end{array}$ & 120 & & & \\
\hline 7 & $2.08-2.35$ & $\begin{array}{l}\text { Infravermelho } \\
\text { Médio }\end{array}$ & 30 & & & \\
\hline
\end{tabular}

Fonte: Embrapa (2012) e Short (2012)

Após a aquisição das imagens, as mesmas foram corrigidas geometricamente. Foram utilizados quarenta pontos de controle espalhados homogeneamente pela imagem, com erro da correção sempre abaixo de um pixel, ou 30 metros, procedimento realizado com o auxílio do módulo OrthoEngine do software PCI Geomatica V10.1 (PCI, 2007). A base cartográfica de referência para o procedimento de correção geométrica foi uma imagem ortorretificada do satélite Landsat 7, sensor $\mathrm{ETM}^{+}$, de 5 de dezembro de 2000, da órbita 223 e ponto 76, produto obtido por download no website da NASA.

\section{Território de pesquisa}

O território de pesquisa foi a microrregião de Paranavaí, no Estado do Paraná (Figura 1). Os municípios selecionados para os trabalhos de campo foram: Alto Paraná, Mirador, Paranavaí, Loanda e Santa Mônica, todos inseridos na microrregião de Paranavaí.

Paranavaí é o município que mais produz mandioca no Estado do Paraná, e muitas propriedades dos municípios próximos a Paranavaí oferecem matéria-prima para as indústrias. A mesorregião do Noroeste Paranaense é a principal região produtora de amido de mandioca do Estado do Paraná e, em consequência, do Brasil. 


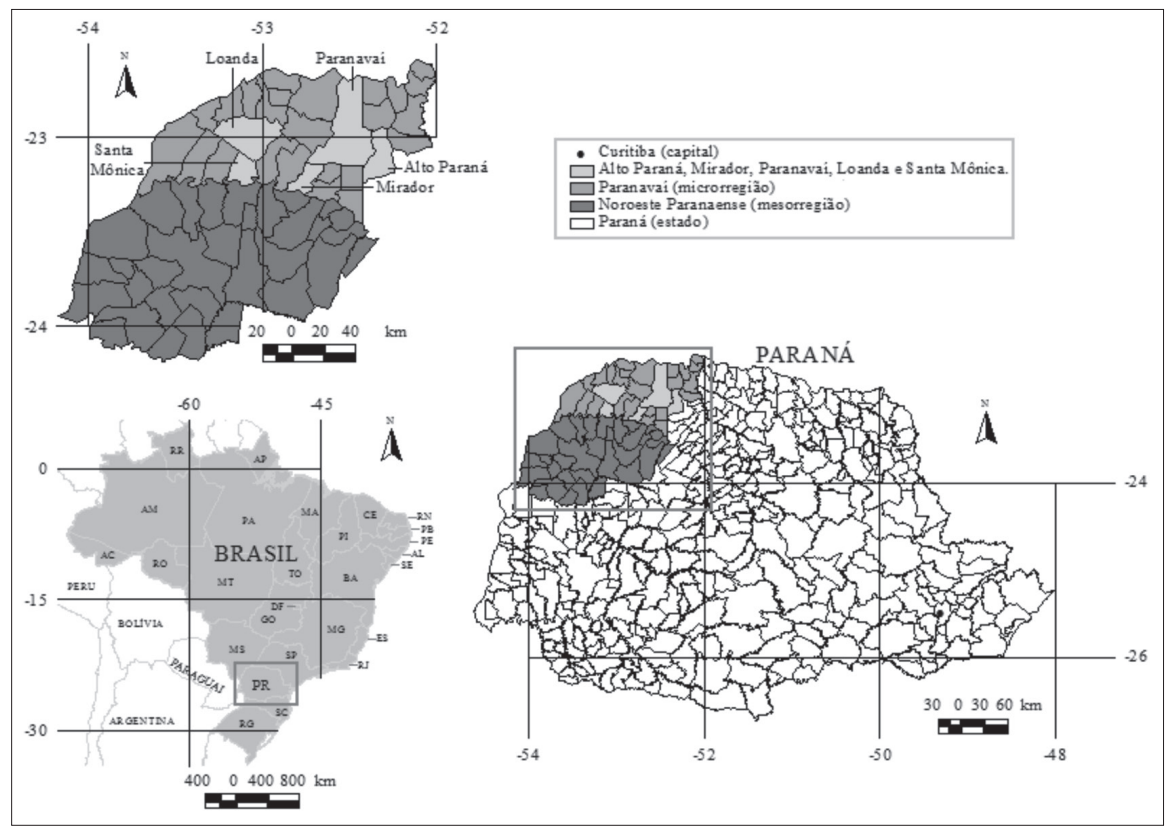

Figura 1 - Localização da região pesquisada.

Avaliação da área cultivada com mandioca

A avaliação da área plantada de mandioca na região de Paranavaí foi dividida em três etapas, começando com a localização de algumas áreas de plantio e elaboração de uma chave de identificação.

\section{a) Levantamento inicial de campo}

Em julho de 2011, foi realizado o primeiro levantamento de campo com a finalidade de georreferenciar alguns cultivos de mandioca com diferentes datas de plantio. Foram georreferenciadas doze áreas em cinco municípios: Alto Paraná (3), Mirador (1), Paranavaí (2), Loanda (2) e Santa Mônica (4). Os plantios de mandioca selecionados foram de várias fases de crescimento da planta: recém-plantados, de primeiro ciclo (10 a 12 meses de idade) e de segundo ciclo (12 a 18 meses). O georreferenciamento foi executado por GPS receptor da marca Garmin, modelo etrex VISTA HCx. 
Foram anotadas informações sobre época de plantio e variedade. Essas informações ajudaram a produzir a chave de identificação do plantio de mandioca por imagens de satélite do Landsat 5 TM.

O software Quantum GIS 1.7.0-Wroclaw transformou as coordenadas do georreferenciamento em arquivos vetoriais na extensão shp. Em seguida, os dados foram exportados para o software PCI Geomatica V10.1, no ambiente de visualização de processamento de dados espaciais do módulo Geomatica Focus (PCI, 2007). Essa operação permitiu visualizar as imagens e ofereceu ferramentas de geoprocessamento para delimitação e identificação dos perímetros de cultivo de mandioca.

No software PCI Geomatica V10.1 e no seu módulo Geomatica Focus (PCI, 2007) foi identificada a resposta espectral do plantio de mandioca ao longo do seu ciclo de desenvolvimento. Para essa identificação foram utilizadas as nove imagens do sensor TM do satélite Landsat 5 descritas anteriormente.

\section{b) Verificação da eficiência do levantamento}

Antes de se utilizar a chave de identificação para estimar a área plantada de mandioca industrial é necessário verificar a precisão dos resultados. Para isso, foi realizada uma verificação no campo, em novembro de 2011. Por meio da chave de identificação, do software PCI Geomatica V10.1 e de seu módulo Geomatica Focus (PCI, 2007), foram classificadas 170 amostras, sendo 85 de plantio de mandioca e 85 de áreas cultivadas com outras culturas. As 170 amostras foram selecionadas nos cinco municípios visitados no primeiro trabalho de campo: Alto Paraná (62), Loanda (44), Mirador (12), Paranavaí (24) e Santa Mônica (28). Em cada município tomou-se o cuidado de identificar metade das áreas com mandioca e a outra metade com outro tipo de cultura. As áreas visitadas na primeira etapa da pesquisa foram excluídas da análise. As amostras foram selecionadas de forma a abranger toda a extensão dos municípios de modo homogêneo.

Para validar a chave de identificação e a estimativa de área, foi criado o modelo de análise apresentado na Figura 2. Esse modelo considera o risco de identificar uma área como sendo ocupada por mandioca, enquanto é de fato ocupada por outra cultura, pastagem ou floresta. Esse risco, que superestima a área plantada com mandioca, foi definido como erro $\beta$, ou erro de tipo II, em analogia com a linguagem adotada em estatís- 
tica. Outro risco avaliado, o erro $\alpha$, ou erro de tipo I, foi considerado como o risco de estimar uma área plantada com mandioca como sendo ocupada por outra cultura. $\mathrm{O}$ erro $\alpha$ leva a subestimar a área real com mandioca.

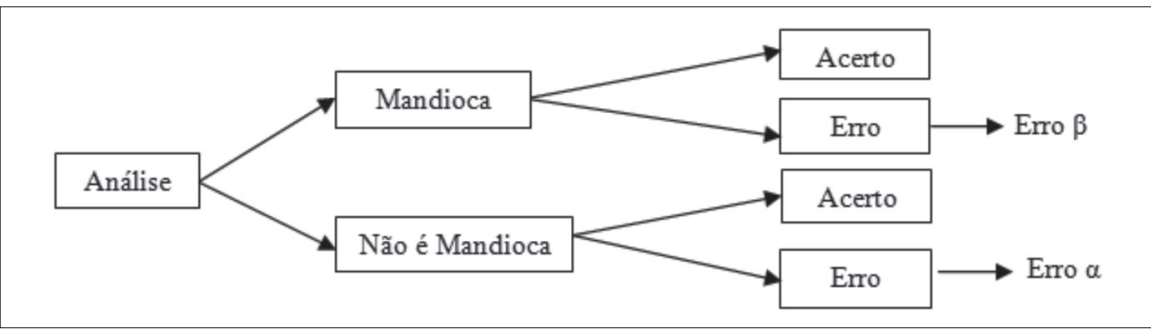

Figura 2 - Modelo de análise utilizado para verificar a precisão da estimativa de área plantada com mandioca

c) Estimativa da área com cultivo de mandioca em três municípios da microrregião de Paranavaí

Entre os cinco municípios visitados no levantamento de campo, três deles, Paranavaí, Mirador e Santa Mônica, foram selecionados para a realização de uma estimativa de área total de mandioca plantada.

A partir do software PCI Geomatica V10.1 e de seu módulo Geomatica Focus (PCI, 2007), foram classificadas visualmente as áreas com plantio de mandioca, trabalho que utilizou as ferramentas de seleção para delimitar os polígonos desse plantio. As imagens do sensor TM/Landsat 5 foram utilizadas para aplicar a chave de classificação elaborada. Os resultados dessa classificação foram comparados com os resultados de estimação do IBGE para os municípios avaliados.

\section{Resultados}

\section{Elaboração da chave de identificação}

A chave de identificação permite identificar as áreas plantadas com mandioca. Como a cultura possui respostas espectrais diferentes em função da fase de desenvolvimento, foi necessário diferenciar os plantios de mandioca entre primeiro e segundo ciclo e as áreas recém-plantadas (Figura 3). 
A primeira classe (Figura 3a) é referente à mandioca de primeiro ciclo na banda TM 4, que representa o infravermelho próximo. Ela se apresenta em cinza médio e, em algumas partes, cinza escuro. A segunda classe (Figura 3b) é a mandioca de segundo ciclo, que é identificada na cor cinza escuro uniforme e sem rugosidade. A terceira classe (Figura 3c) é um plantio de mandioca com menos de quatro meses. Esse plantio pode ser identificado pela tonalidade escura.

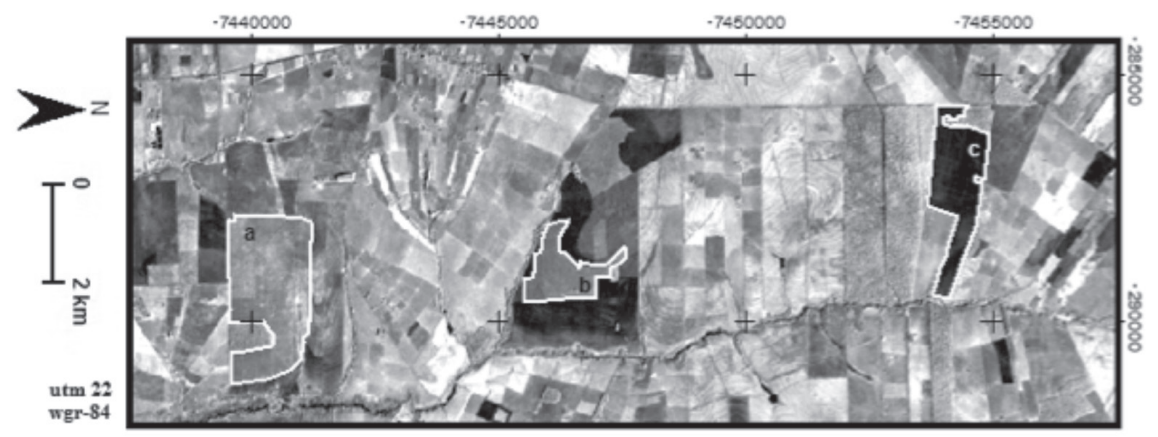

Legenda:

a) cultivo de mandioca de primeiro ciclo;

b) cultivo de mandioca de segundo ciclo;

c) mandioca com menos de quatro meses.

Figura 3 - Banda individual da imagem TM/Landsat de uma porção dos municípios de Santa Mônica e de Loanda, no Estado do Paraná. Obtida em 4 de junho de 2011

Banda TM4 na resolução de $30 \mathrm{~m} \times 30 \mathrm{~m}$.

Para facilitar a análise, as áreas com plantio de mandioca foram agrupadas independentemente da idade da cultura. A análise permitiu a identificação de áreas acima de 2 ha. Essa limitação não prejudicou a análise, pois as áreas menores de plantação de mandioca na região são de um alqueire, ou seja, 2,4 ha.

\section{Verificação da eficiência da chave de identificação}

Após a elaboração da chave de identificação, foi realizada a verificação das estimativas conforme abordado na Metodologia. Os resultados estão apresentados na Tabela 2. 
Tabela 2 - Verificação da eficiência das estimativas feitas a partir da chave de identificação.

\begin{tabular}{|l|c|c|c|c|c|}
\hline Municípios & $\mathrm{N}^{\circ}$ de amostras & $\begin{array}{c}\text { Amostras realmente } \\
\text { com mandioca }\end{array}$ & $\begin{array}{c}\text { Erro } \beta \\
(\%)\end{array}$ & $\begin{array}{c}\text { Amostras realmente } \\
\text { sem mandioca }\end{array}$ & $\begin{array}{c}\text { Erro } \alpha \\
(\%)\end{array}$ \\
\hline Alto Paraná & 31 & 30 & 3,2 & 30 & 3,23 \\
\hline Loanda & 22 & 20 & 9,1 & 22 & 0,0 \\
\hline Mirador & 6 & 6 & 0,0 & 6 & 0,0 \\
\hline Paranavaí & 12 & 11 & 8,3 & 12 & 0,0 \\
\hline Santa Mônica & 14 & 14 & 0,0 & 14 & 0,0 \\
\hline & 85 & 81 & 4,7 & 84 & 1,2 \\
\hline
\end{tabular}

O erro de superestimação da área plantada, ou erro $\beta$, foi na média de $4,7 \%$, o que pode ser considerado baixo. Os resultados do modelo de análise para o erro $\alpha$, que subestima a avaliação, foram ainda mais satisfatórios, com apenas uma área em Alto Paraná, que tinha mandioca e foi considerada como ocupada por outra cultura. Como os dois tipos de erro possuem efeitos opostos, eles tendem a se neutralizar. Assim, a subestimação de 1,2\% permite reduzir a superestimação calculada, com um resultado final que superestima a realidade numa percentagem inferior a $4,7 \%$. Para efeito de cálculo, o erro final foi obtido da subtração do erro $\alpha$ ao erro $\beta$, ou seja, $3,5 \%$.

O tamanho médio das áreas plantadas com mandioca, em hectares, a partir das amostras selecionadas e confirmadas, foi de 38,85 ha no município de Loanda; 46,83 ha em Alto Paraná; 49,70 ha em Mirador; 69,57 ha em Paranavaí; e 118,15 ha em Santa Mônica. A média geral de área plantada com mandioca foi de 64,69 hectares.

Estimativa da área total plantada com mandioca em três municípios, na microrregião de Paranavaí

A estimativa de área total plantada com mandioca para os municípios de Mirador, Paranavaí e Santa Mônica foi, respectivamente, de 579,8 ha; 3153,6 ha; e 2082,5 ha. Em junho de 2011, as estimativas de área plantada, segundo o IBGE, apresentaram os seguintes valores para os mesmos municípios: 1261 ha (Mirador); 5490 ha (Paranavaí); e 1700 ha (Santa Mônica) - conforme Tabela 3. Em Mirador e Paranavaí, a análise por georreferenciamento subestimou a área plantada com mandioca, em relação aos dados do IBGE, ${ }^{1}$ em 54\% e 43\%. Em Santa Mônica ocorreu uma superestimação em $22 \%$. 
Tabela 3 - Comparação das áreas estimadas por geotecnologias com as estimações do IBGE, no ano de 2011

\begin{tabular}{|l|c|c|c|}
\hline \multirow{2}{*}{ Municípios } & \multicolumn{2}{|c|}{ Comparação (ha) } & \multirow{2}{*}{$\begin{array}{c}\text { Medida / IBGE } \\
(\%)\end{array}$} \\
\cline { 2 - 3 } & Área medida & Área estimada (IBGE) & -54 \\
\hline Mirador & 580 & 1261 & -43 \\
\hline Paranavaí & 3154 & 5490 & +22 \\
\hline Santa Mônica & 2082 & 1700 & \\
\hline
\end{tabular}

O levantamento da Produção Agrícola Municipal (PAM), realizado pelo IBGE, fornece informações sobre as áreas de lavouras, produção e rendimento médio. A coleta de informações é realizada por intermédio da aplicação de um questionário em cada município do país. O preenchimento é feito pelo agente de coleta do IBGE. As estimativas realizadas pelos agentes resultam de contatos que os mesmos mantêm com técnicos do setor agrícola, com grandes produtores, ou pelo conhecimento que os agentes possuem sobre as atividades agrícolas da sua área de atuação (IBGE, 2002, p. 8).

A diferença entre os resultados da pesquisa e do PAM justifica-se pelas diferenças entre os métodos realizados. No caso da mandioca, a grande quantidade de produtores e a grande variação de área plantada, de um ano para outro, dificultam a obtenção de informações detalhadas a partir de um levantamento de campo, como o realizado pelo IBGE. Já as imagens do sensor TM/Landsat 5 são informações reais, regulares e que necessitam de um analista experiente para interpretação. Essas imagens permitem verificar toda a extensão municipal, com custo reduzido e possibilidade de atualização mensal.

\section{Considerações finais}

A pesquisa comprovou a possibilidade de se utilizarem imagens de satélites do sensor TM/Landsat 5 para estimar as áreas com plantio de mandioca e oferecer informações com precisão. Os dados da pesquisa possuem precisão acima de $95 \%$. As imagens do sensor TM/Landsat 5 poderiam ser utilizadas para estimar as áreas com cultura da mandioca e melhorar as informações fornecidas ao setor, e, conforme já sugerido por Rizzi e Rudorff (2005, p. 226), poderiam ser utilizadas para um correto mapeamento da soja. 
Os resultados obtidos na pesquisa deverão ser complementados para maximizar o potencial desse recurso, como a automatização da análise realizada por classificação semiautomática ou classificação automática. A classificação manual pode ser aplicada em alguns municípios ou porção territorial, mas a aplicação em escala territorial maior, como o Estado do Paraná, torna a avaliação muito demorada.

As indústrias feculeiras podem utilizar a classificação manual para levantar informações essenciais para suas transações comerciais, como a quantidade de áreas disponíveis, o período e a localização de plantio, entre outras informações. Esses dados permitirão às empresas antecipar a safra e corrigir futuras distorções.

A aplicação desta pesquisa poderá trazer um impacto para a agricultura familiar, principalmente para os produtores de mandioca industrial com áreas superiores a 2,5 ha, localizadas essencialmente nos Estados do Paraná, São Paulo e Mato Grosso do Sul. As informações de safra favorecem a estabilidade do setor e facilitam o planejamento dos produtores. No entanto, é importante verificar a boa divulgação das informações e cuidar para que os dados não fiquem nas mãos das empresas, que poderiam se beneficiar de informações privilegiadas para manipular os preços.

$\mathrm{O}$ uso das imagens de satélites como as do sensor TM/Landsat 5 poderá ser inviável para regióes brasileiras onde as áreas cultivadas com mandioca são inferiores a 1 ha, como no Norte e Nordeste, principais regiões produtoras de mandioca no país, com destino essencialmente para produção de farinha. Os resultados da pesquisa indicam uma boa identificação apenas com plantios acima de 2 ha.

Seria interessante testar também essa metodologia em outras regiões produtoras de mandioca industrial para fecularias, como o oeste do Estado do Paraná, o sul de Mato Grosso do Sul e o sudeste do Estado de São Paulo. Essas regiões possuem perfis diferentes da região de Paranavaí, com outras variedades e tipos diferentes de agricultura, especialmente o oeste do Paraná, que confere maior importância à agricultura realizada em pequenas explorações familiares. No entanto, essas produções são importantes e podem influenciar os preços nas empresas em Paranavaí. Uma previsão completa da produção de mandioca para o setor de fécula depende de uma previsão eficiente em todas essas regiões. 


\section{Agradecimentos}

Os autores agradecem a Paulo Boni, da Yoki Alimentos S/A, e a Vanderlei Colussi, da Agroindustrial Paranaense de Polvilho Ltda., pelo apoio nos levantamentos de campo.

\section{Nota}

1. Informações pessoais fornecidas pelo IBGE - Unidade Estadual do Paraná.

\section{Referências}

ABAM - Associação Brasileira dos Produtores de Amido de Mandioca. Produção brasileira de amido - por Estado. Paranavaí, 2012. Disponível em: <www.abam. com.br>. Acesso em: 16 nov. 12.

ADAMI, M.; MOREIRA, M. A.; RUDORFF, B. F. T.; FREITAS, C. da C.; FARIA, R. T. de. Expansão direta na estimativa de culturas agrícolas por meio de segmentos regulares. Revista Brasileira de Cartografia, Brasília, v. 1, n. 57. p. 22-27, 2005.

BURROUGH, P. A. Principles of Geographical Information Systems for Land Resources Assessment. Oxford: Oxford University Press, 1986. 194 p.

CÂMARA, G.; MEDEIROS, J. S. Princípios básicos em geoprocessamento. In: ASSAD, E. D.; SANO, E. E. Sistema de informações geográficas. Aplicações na agricultura. Brasília: Embrapa-SPI/Embrapa-CPAC, 1998. cap. 1, p. 3-11.

CARDOSO, C. E. L.; BARROS, G. S. C. Competitividade no segmento agrícola da cadeia de fécula de mandioca. In: Congresso Brasileiro de Economia e Sociologia Rural, 42, 2004, Cuiabá. Anais ... Rio de Janeiro: Sober, 2004. p. 1-21.

CARDOSO, C. E. L. Competitividade e inovação tecnológica na cadeia agroindustrial de fécula de mandioca no Brasil. Tese (Doutorado em Ciências) - Escola Superior de Agricultura Luiz de Queiroz, Piracicaba, 2003.

CHAO, Z.; XIAODONG, Z.; JIANYU, Y.; HAIXIA, L.; DEHAI, Z.; WANBIN, Z. Evaluation of cassava planting potential with remote sensing and GIS. New Zealand Journal of Agricultural Research, New Zealand, v. 50, n. 5, p. 1135-1140, 2007.

CIRANI, C. B. S.; MORAES, M. A. F. D. Inovação na indústria sucroalcooleira paulista: os determinantes da adoção das tecnologias de agricultura de precisão. Revista de economia e sociologia rural, Brasília, v. 48, n. 4, p. 543-565, 2011.

EIUMNOH. A.; SHRESTHA, R. P. A Study on Estimation of Cassava Area and Production Using Remote Sensing and Geographic Information Systems in the Northeast Region of Thailand. Southeast Asian Studies, v. 37, n. 3, p. 417-430, 1999.

EMBRAPA - Empresa Brasileira de Pesquisa Agropecuária. LANDSAT - Land Remote Sensing Satellite. Disponível em: <http://www.sat.cnpm.embrapa.br/ conteudo/landsat.htm>. Acesso em: 5 jan. 2012. 
FAO - Food and Agriculture Organization of the United Nations. The world cassava economy: facts, trends and outlook. Rome: FAO \& IFAD, 2000. p. 64. Disponível em: <http://www.fao.org/docrep/009/x4007e/x4007e00.htm>. Acesso em: 14 jul. 2012.

FAOSTAT - Food and Agriculture Organization of the United Nations. Production. Roma, 2012. Disponível em: <http://faostat.fao.org/>. Acesso em: 24 jan. 12.

FELIPE, I. F.; ALVES, L. R. A.; CARDOSO, C. E. L.; GEROTO, C. L. Organização e coordenação na indústria de fécula de mandioca no Brasil sob a ótica da economia dos custos de transação. In: Congresso da Sociedade Brasileira de Economia, Administração e Sociologia Rural, 48, 2010, Campo Grande. Anais eletrônicos... Brasília: SOBER, 2010. Disponível em: <http://www.sober.org.br/>. Acesso em: 6 fev. 2011. p. 1-17.

FONTANA, D. C.; WEBER, E.; DUCATI, J. R.; FIGUEIREDO, D. C.; BERGAMASCHI, H.; BERLATO, M. A. Monitoramento e previsão de safras no Brasil. [CDROM]. In: Simpósio Latino Americano de Percepción Remota, 9, 2000. Anais... Puerto Iiguazú, 2000. p. 1-11.

GOEBEL, M. A. Organização e coordenação do sistema agroindustrial da mandioca na microrregião Oeste do Paraná. Dissertação (Mestrado em Desenvolvimento Regional e Agronegócio) - UNOESTE, Toledo, 2005.

IBGE - Instituto Brasileiro de Geografia e Estatística. Pesquisas agropecuárias. Rio de Janeiro: IBGE, 2002. 92 p.

NOGUEIRA, A. C. L. Risk perception and management for cassava starch producers in Brazil. In: Conference of International Food and Agribusiness Management Association, 9, 1999. Anais... Florença: IAMA, 1999.

NOVO, E. M. L. Sensoriamento remoto: princípios e aplicações. 4. ed. São Paulo: Blucher, 2011. 388p.

PCI. Geomatics.Versão 10.1 for Windows. Ontário, Canadá, 2007.

RAMIREZ, M. R.; SOUZA, J. M. Sistema gerenciador de banco de dados em sistemas de informações geográficas. In: MEIRELLES, M. S. P.; CÂMARA, G.; ALMEIDA, C. M. Geomática: modelos e aplicações ambientais. Brasília: Embrapa Informação Tecnológica, 2007. p. 55-103.

RINALDI, R. N.; ROCHA JUNIOR, W. F.; SOUZA, E. F.; STADUTO, J. A. R. A cultura da mandioca na região Oeste do Paraná: um estudo da coordenação da cadeia sob a ótica da teoria dos contratos. Organizações Rurais e Agroindustriais, v. 7, n. 1, p. 11-22, 2005.

RIZZI, R.; RUDORFF, B. F. T. Estimativa da área de soja no Rio Grande do Sul por meio de imagens Landsat. Revista Brasileira de Cartografia, v. 3, n. 57, p. 226-234, 2005.

RUDORFF, B. F. T.; BERKA, L. M. S.; XAVIER, A. C.; MOREIRA, M. A.; DUARTE, V.; ROSA, V. G. C.; SHIMABUKURO, Y. E. Estimativa de área plantada com canade-açúcar em municípios do Estado de São Paulo, por meio de imagens de satélites e técnicas de geoprocessamento, safra 2003/2004. Instituto Nacional de Pesquisas Espaciais (INPE). São José dos Campos, 2004. INPE - 10791- RPQ/759. 54p. 
SANCHES, I. D.; EPIPHANIO, J. C. N.; FORMAGGIO, A. R. Culturas agrícolas em imagens multitemporais dos satélites Landsat. Agricultura em São Paulo, v. 52, n. 1, p. 83-96, 2005.

SANO, E. E.; ASSAD, E. D.; ORIOLI, Á. L. Monitoramento da ocupação agrícola. In: ASSAD, E. D.; SANO, E. E. Sistema de informações geográficas. Aplicações na agricultura. Brasília: Embrapa-SPI/Embrapa-CPAC, 1998. cap. 1, p. 179-190.

SANTOS, A. O.; VAlERIANO, M. M.; MAZIERO, J. V. G.; CAVALLI, A. C. Opportunities and limitations for the application of simulation and modeling as a support for precision farming. Acta Scientiarum, Maringá, v. 23, n. 6, p. 15091517, 2001.

SHORT. Tutorials. 2012. Disponível em: <http://rst.gsfc.nasa.gov/>. Acesso em: jan. 2012.

VILPOUX, O. F. Competitividade da mandioca no Brasil como matéria-prima para amido. Informações Econômicas, São Paulo, v. 38, n. 11, p. 27-38, nov. 2008.

VILPOUX, O. F. Arranjos institucionais nas transações entre produtores e fecularias de mandioca: abordagem pela economia dos custos de transação. Informe Gepec, Toledo, v. 14, n. 1, p. 127-146, 2010.

Brychtn Ribeiro de Vasconcelos - Graduado em Geografia pela Universidade Católica Dom Bosco - Mestre em Desenvolvimento Local pela mesma universidade.

Olivier François Vilpoux - Graduado em Engenharia Agronômica pelo Institut Supérieur D'agriculture, na França - Mestre em Administração pelo Institut de Gestion Internationale en Agro Alimentaire - Doutor em Engenharia de Produção pelo Institut National Polythecnique de Lorraine - Professor pesquisador da Universidade Católica Dom Bosco, Campo Grande, Mato Grosso do Sul.

Antonio Conceição Paranhos Filho - Graduado em Geologia pela Universidade Federal do Paraná - Mestre e Doutor em Geologia pela mesma Universidade - Professor Associado da Universidade Federal de Mato Grosso do Sul, Campo Grande (MS). 